\title{
The Silicon Tracker of the H1 Detector
}

\author{
Benno List \\ Institute for Particle Physics, ETH Zurich, CH-8093 Zurich ${ }^{1}$
}

\begin{abstract}
The H1 experiment at HERA is equipped with a silicon vertex detector, comprising a barrel part and two endcaps with disks. The barrel part uses double sided, DC coupled strip sensors, whereas the endcap parts use two types of wedge-shaped sensors, both single sided and AC coupled: $u / v$-sensors have strips parallel to one edge of the sensor, $r$-sensors have circular strips. Additional pad detectors provide fast triggering signals in the backward part.
\end{abstract}

Key words: Silicon vertex detector PACS: 29.40.Gx, 29.40.Wk

\section{Introduction}

The H1 detector [1] at the ep collider HERA at DESY has been in operation since 1992. In 1995 it was equipped with a central (CST) and backward (BST) silicon tracker (the direction of the proton beam defines the "forward" direction at H1). During the luminosity upgrade of HERA in 2001, the CST and BST were modified, and a forward silicon tracker (FST) was added. The complete silicon vertex detector consists of a barrel part, the CST, with two layers of double-sided silicon strip detectors, and the two disk detectors BST and FST that use single-sided strip detectors, plus pad detectors for triggering purposes in the back (BST-Pad). In 2004, the FST was damaged due to a water leak, and underwent, together with the BST, extensive repair work during the 2005 running period, so that H1 operated only with the CST during that time. In December 2005, both detectors were successfully re-installed in the $\mathrm{H} 1$ detector.

\footnotetext{
Email address: Benno.List@desy.de (Benno List).

URL: http://www-h1.desy.de/ blist (Benno List).

1 Supported by the Swiss National Science Foundation
} 


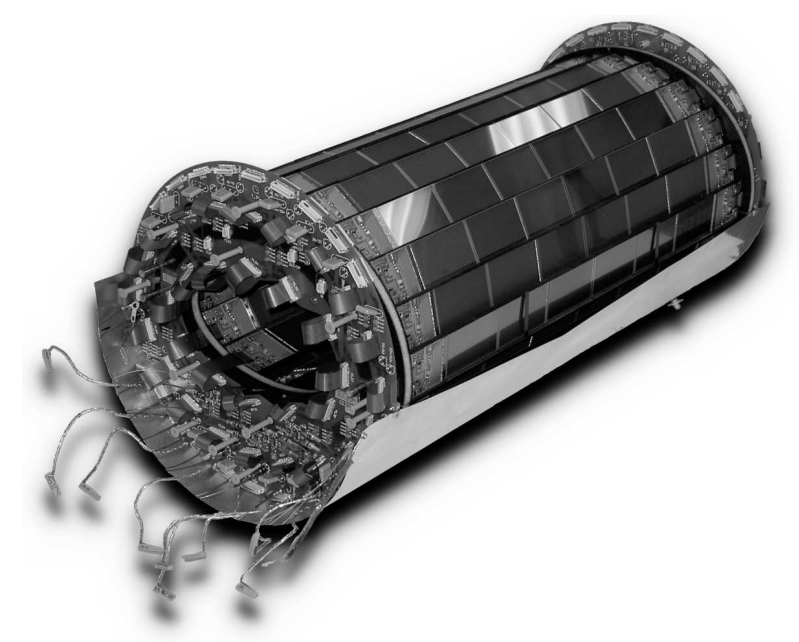

Fig. 1. The H1 central silicon tracker.

\section{Strip Detectors}

While the subdetectors of the H1 silicon tracker are equipped with strip detectors of different designs, their readout follows a common scheme, using the APC128 readout chip developed at the PSI with an analog data transmission to the electronics trailer, where the signals are digitized and processed.

\subsection{The Central Silicon Tracker}

The CST [2] (Fig. 1), built in a collaboration of PSI, ETH and University of Zurich, uses double-sided, DC-coupled strip sensors [3] with a size of $5.9 \times$ $3.4 \mathrm{~cm}^{2}$. The p side has strips parallel to the $z$ axis with a readout (strip) pitch of $50 \mu \mathrm{m}(25 \mu \mathrm{m})$. The $\mathrm{n}$ side measures the $z$ coordinate with a strip pitch of $88 \mu \mathrm{m}$, without any intermediate strips; a second metal layer contains the readout lines. Six sensors are daisy-chained to form a ladder, with a readout hybrid for $2 \times 640$ channels ( $\mathrm{p}$ and $\mathrm{n}$ side) at either end. 32 ladders are used, 12 in the inner and 20 in the outer layer, with a total of 81920 readout channels.

The CST was installed starting in 1995 and fully operational in 1997. During the luminosity upgrade in 2000/01, the readout hybrids were replaced by new hybrids with radiation-hard readout chips (see below). The mechanical arrangement of the ladders was changed to accomodate the new, elliptical $\mathrm{BeAl}$ beam pipe made necessary by the changes to the HERA storage ring [4]. In the years 1997 to 2000, a signal over noise of 19 for the p side and 7 for the $\mathrm{n}$ side has been measured; the asymptotic impact parameter resolution was $57 \mu \mathrm{m}$. The new data indicate that the new DMILL version of the APC readout chip achieves a similar signal over noise ratio, as expected from laboratory measurements [5]. 


\subsubsection{CST Movements between Beams}

The CST operation in 2005 was hampered by the fact that the H1 detector is positioned approximately $4 \mathrm{~mm}$ too low with respect to the HERA beampipe. While this caused no problems in 2004, in the 2005 running period the CST actually touched the beampipe.

The superconducting final focus magnets, which were newly installed within the $\mathrm{H} 1$ solenoidal field in 2000, experience large magnetic forces up to $8 \mathrm{kN}$ that result in a vertical movement of these magnets. Although the central part of the beampipe is mechanically decoupled by bellows from these magnets, a vertical movement of the beampipe by approximately $1.5 \mathrm{~mm}$ was observed in 2005 when the magnets were ramped up to the field strength necessary for luminosity operation. This caused a vertical movement of the upper half shell of the CST by about $1.5 \mathrm{~mm}$ at the beginning of each injection cycle. Therefore, he exact CST position varies from fill to fill. Typically, these variations are smaller than $20 \mu \mathrm{m}$, but they can be as large as $100 \mu \mathrm{m}$.

These movements made it necessary to perform an alignment of the CST's position with respect to the $\mathrm{H} 1$ coordinate system for each individual luminosity fill. In 2005, alignment constants for 211 such fills were determined, separately for the top and bottom halves of the CST. The alignment was based on tracks from cosmic muons recorded during $e p$ data taking, and tracks from $e p$ interactions with transverse momenta above $1.5 \mathrm{GeV}$. Both subsamples have about equal weights in the alignment procedure. The typical position errors are presently around $20 \mu \mathrm{m}$ for each half shell, depending on the length of the luminosity fill.

\subsection{The Forward Silicon Tracker}

The FST [7], built by a collaboration of DESY-Zeuthen and Charles University Prague, was first installed after the upgrade in 2001. It consists of several disks with twelve sensors each, covering three quarters of the azimuthal angle. One quarter is not covered due to space restrictions caused by the elliptical beampipe.

All sensors are wedge-shaped, with an inner (outer) radius of the sensitive area of $59(120) \mathrm{mm}$, cover $22.5^{\circ}$ of azimuthal angle, and have $640 \mathrm{AC}-$ coupled readout strips on the $\mathrm{p}$-side, while the $\mathrm{n}$-side is not read out. Two different sensor types are used: There are so-called $r$-sensors, whose 640 readout strips form concentric arcs with a readout pitch of $96 \mu \mathrm{m}$ measuring the $r$ coordinate; a second metal layer provides readout lines running to the outer edge of the sensor, where they are connected to the readout hybrid. In addition, there are $u$ or $v$-sensors (the designation depends on the orientation of the sensor 
in the detector), which have 640 strips at a readout pitch of $75 \mu \mathrm{m}$, running parallel to one edge of the wedge. These sensors have only one metal layer and consequently a smaller interstrip capacitance that leads to a better signal-tonoise ratio of 30, compared to 15 for the $r$-sensors.

In the configuration of 2001-2004, Five $u / v$ wheels were used, where pairs of $u$ and $v$-sensors were mounted back-to-back, resulting in a $22.5^{\circ}$ stereo angle. These were interspersed with two wheels of $r$-sensors. This configuration had 92160 readout channels. The resolution after alignment, as determined from the triplet sagitta, was $12 \mu \mathrm{m}$.

In early 2004, the FST was damaged by a water leak in its cooling circuitry. The water lead to an increased humidity within the detector volume, causing condensation on the readout hybrids, which as a result were damaged beyond repair. The FST was therefore removed from the H1 detector during the shutdown in autumn 2004 and completely rebuilt. In order to make the FST more tolerant against radiation damage after the refurbishment, a new, radiation hard version of the APC readout chip was developed and used.

In the new configuration, the FST comprises five $u / v$ wheels, giving a total of 76800 readout channels. Due to time and budgetary restrictions, the damaged $r$-sensors were not replaced. In December 2005, the new FST was successfully installed in the $\mathrm{H} 1$ detector.

\subsection{The Backward Silicon Tracker}

The BST [8], built by the same institutes as the FST, was first installed in the H1 detector in 1995. Since then, it underwent a series of modifications and upgrades [9]. For HERA-II, the BST initially consisted of eight wheels with $r$-sensors, along with four wheels of the BST-Pad detector that is described below.

After considerable radiation damage to the readout chips in $2002 / 03$, all $r$ sensors had to be removed in 2003 and were replaced by six wheels with $u$ and $v$-sensors (in one wheel, only $u$-sensors were installed due to a lack of available readout chips). Due to the relatively low multiplicities typically observed in the backward direction, the $r$-sensors were not considered to be necessary anymore, in particular in view of the superior signal to noise ratio of the $u / v$-sensors. This configuration had a total of 84480 readout channels.

After the accident that damaged the FST in 2004, it was decided to rebuild the BST together with the FST, in order to ensure that the detector will be fully functional for the final running period of HERA-II in 2006/07. Therefore, the BST was removed in autumn 2004 and completely rebuilt. The new 
configuration has six $u / v$ wheels, giving a total of 92160 readout channels. It was successfully installed in December 2005.

\subsection{Read Out and Signal Processing}

The BST, CST, and FST strip detectors are read out with chips of the APC128 family that was originally developed at the Paul-Scherrer-Institute [10]. The CST has been upgraded in 2001 and 2003 to the radiation-hard version APC128D [5] fabricated in DMILL [6] technology. The BST and FST were upgraded in 2005 to a new, radiation hard version of the APC readout chip and an accompanying decoder chip that were developed in a collaboration of DESY (Zeuthen), the Kirchhoff Institute for Physics of the University of Heidelberg, and the PSI. These chips are produced in $0.25 \mu \mathrm{m}$ CMOS technology by UMC [11]. The translation of the existing chip designs to the new technology could be achieved by a single person within only three months.

The APC128(D) provides a preamplifier and a 32 stage analog pipeline for 128 channels. These chips are located on readout hybrids attached to the sensors, with their inputs directly bonded to the sensor readout lines. Analog addition and subtraction of signals from different pipeline stages can be carried out on the chip, a feature that is used for pedestal subtraction. After a Level-2 trigger decision, the signals from ten APC chips on two neighboring hybrids are read out sequentially at a frequency of about $1.5 \mathrm{MHz}$. This is steered by four digital signals generated by sequencer units designed by DESY, the so-called OnSiRoCs [12], that are located in the electronics trailer. While the BST and FST use copper cables for the signal transmission from the front-end hybrids to the electronics trailer, the CST employs an optical data transmission of the steering signals and the analog readout signals [13].

Digitization and further processing take place in the electronics trailer, in a farm of PowerPC based processor boards [14]. Each processor board is equipped with a custom mezzanine board, designed by RAL, that contains eight FADCs, buffer memory, and control logic, and processes data from eight analog input lines, i. e. 10240 channels. This data is transferred via PCI bus into the processor's memory, which then performs common mode correction, pedestal subtraction and hit identification, and sends the sparsified data to the $\mathrm{H} 1$ data aquisition after a positive Level-3 trigger decision. 


\section{The BST-Pad trigger device}

While the hit information of the silicon strip detectors is only available after a Level-3 trigger decision, the BST-pad detector [15] has been designed to provide fast signals as input for the Level-1 trigger within $2.5 \mu \mathrm{s}$. 4 wheels equipped with pad sensors have been installed to that end.

The AC-coupled pad sensors have the same dimensions as the other sensors employed in the BST. Each sensor is segmented into 32 pads with areas of 33 to $116 \mathrm{~mm}^{2}$. The $\mathrm{PRO} / \mathrm{A}$ readout chip, an ASIC developed by Ideas ASA (Oslo) [16], is used for the hit detection, which has to be performed at the bunchcrossing frequency of $10.4 \mathrm{MHz}$. The chip contains preamplifiers, shapers, and discriminators for 32 channels and provides common mode suppression by the subtraction of signals between neighboring channels.

An additional readout board within the detector volume processes the hit data with complex programmable logic devices (CPLDs) from ALTERA [17] to identify tracks, and provides slow control functionality. The output signals of the trigger boards are transferred to the trailer, where they are combined and Level-1 trigger signals are generated. Additional information on the direction of observed tracks is transferred to the Level-2 trigger logic.

\section{Acknowledgments}

I would like to thank Daniel Pitzl, Peter Kostka, Ilya Tsurin, Mirek Nozicka, Hans Henschel, Guillaume Leibenguth and Tobias Zimmermann for their valuable help in the preparation of this talk, and the good cooperation in the operation of the $\mathrm{H} 1$ silicon tracking devices.

\section{References}

[1] I. Abt et al. [H1 Collaboration], Nucl. Instrum. Meth. A 386 (1997) 310347, ibid. 348-396.

[2] D. Pitzl et al., Nucl. Instrum. Meth. A 454 (2000) 334-349.

[3] D. Pitzl et al., Nucl. Instrum. Meth. A 348 (1994) 454-460.

[4] B. List, Nucl. Instrum. Meth. A 501 (2001) 49-53.

[5] M. Hilgers, R. Horisberger, Nucl. Instrum. Meth. A 481 (2002) 556-565.

[6] M. Dentan et al., IEEE transaction on Nucl. Science, 43 (1996) 1763-1767.

[7] M. Nozicka, Nucl. Instrum. Meth. A 501 (2003) 54-59.

[8] H. Henschel, R. Lahmann, Nucl. Instrum. Meth. A 453 (2000) 93-97.

[9] B. List, Nucl. Instrum. Meth. A 549 (2005) 33. 
[10] R. Horisberger, D. Pitzl, Nucl. Instrum. Meth. A 326 (1993) 92-99.

[11] United Microelectronics Corporation, http://www .umc .com/.

[12] J. Bürger et al., Nucl. Instrum. Meth. A 386 (1997) 269-279.

[13] W. Erdmann et al., Nucl. Instrum. Meth. A 372 (1996) 188-194.

[14] W. J. Haynes et al., Nucl. Instrum. Meth. A 403 (1998) 313.

[15] I. Tsourine, Nucl. Instrum. Meth. A 501 (2003) 219-221.

[16] Ideas ASA, http://www.ideas.no/.

[17] ALTERA, http://www.altera.com/. 